\title{
Environmental Energy Harvesting Techniques to Power Standalone IoT-Equipped Sensor and Its Application in 5G Communication
}

\author{
Satyanand Singh ${ }^{1 *}$ \\ ${ }^{1}$ Assistant Professor, Fiji National University, Suva, Fiji Island
}

\begin{abstract}
In the recent few years, due to its significant deployment to meet global demand for smart cities, the Internet of Things (IoT) has gained a lot of attention. Environment energy harvesting devices, which use ambient energy to generate electricity, could be a viable option in near future for charging or powering stand-alone IoT sensors and electronic devices. The key advantages of such energy harvesting gadgets are that they are environmentally friendly, portable, wireless, cost-effective, and compact. It is significant to propos and fabricate an improved, high-quality, economical, and efficient energy harvesting systems to overcome power supply to tiny IoT devices at the remote locations. In this article, various types of mechanisms for harvesting renewable energies that can power sensor enabled IoT locally, as well as its associated wireless sensor networks (WSNs), are reviewed. These methods are discussed in terms of their advantages and applications, as well as their drawbacks and limitations. Furthermore, methodological performance analysis for the decade 2005 to 2020 is surveyed in order to identify the methods that delivered high output power for each device. Furthermore, the outstanding breakthrough performances of each of the aforementioned micro-power generators during this time period are emphasized. According to the research, thermoelectric modules can convert up to $2500 \times 10^{-3} \mathrm{~W} / \mathrm{cm}^{2}$, thermo-photovoltaic $10.9 \%$, piezoelectric $10,000 \mathrm{~mW} / \mathrm{cm}^{3}$ and microbial fuel cell $6.86 \mathrm{~W} / \mathrm{m}^{2}$ of energy.
\end{abstract}

\section{Keywords:}

Energy Harvesting; IoT; Smart City; WSNs; Machine to Machine; Piezoelectric; Photovoltaic; Solar Energy; Thermal Energy.

\section{Article History:}

Received: 05 August 2021

Revised: 18 October 2021

Accepted: 27 October 2021

Published: 15 November 2021

\section{1- Introduction}

Machine to Machine (M2M) communication with little or no human intervention is becoming more and more common. Applications for M2M communications and the IoT include smart grid automation, healthcare, agricultural systems, transportation systems, industrial production, housing automation, and environmental monitoring. The creation of complex network architectures consisting of interconnected wireless devices that make up deice-to-device (D2D) or more generally, M2M communication networks is a key feature of 5G networks. For example, some IoT-generating appliances, sensors, or portable devices. With the emerging access infrastructure, all these complex network architectures co-exist. Furthermore, without causing congestion problems, the traffic created by various M2M and IoT applications must be properly allocated to the access point. In addition, not all IoT devices [1] can be linked to a grid, so energy harvesting plays a key role in achieving the energy-neutral or permanent operation of a small battery wireless system. Energy harvesting and on-demand energy replenishment of depleted batteries [2] are two main technologies that will allow the IoTs to work sustainably.

For decades, computing technology has been seen as dynamic, adaptable, and tightly connected. Kevin Ashton first proposed the term "IoT" in 1999 [3, 4]. A main technical component of emerging intelligence and security technologies

\footnotetext{
* CONTACT: satyanand.singh@fnu.ac.fj

DOI: http://dx.doi.org/10.28991/esj-2021-SP1-08
}

(C) 2020 by the authors. Licensee ESJ, Italy. This is an open access article under the terms and conditions of the Creative Commons Attribution (CC-BY) license (https://creativecommons.org/licenses/by/4.0/). 
in the computing world is known to be the definition of the IoT. The IoT component architecture is focused on its powerful capabilities for cognition and communication. Wireless devices on the network use IoT-powered sensors and devices to gather statistics, data, and information from the surrounding. The IoTs can be used in several applications like embedded systems [5], safekeeping monitoring systems [6,7], controllers [8,9], transport systems [10, 11], wearable devices [12], energy Tracking [13], environmental monitoring System [14, 15], object tracking [16], traffic monitoring $[17,18]$, intelligent agriculture [19, 20], and human domain networks [21] has clearly increased. By end of 2020 more than twenty-six billion IoT devices are expected to be connected online for commercial purpose. For smart city scenarios, several connections are expected to be much higher, over one hundred billion, some even estimate this figures [22]. All IoT based devices are so small that they require less power to maintain functionality [23]. The IoTs growth can be constrained by the difficulty of obtaining a constant power source to power the system. Most IoTs devices are mounted in remote inaccessible areas with limited use of conventional energy sources, making it adversity to power IoT devices for service [23, 24]. Batteries are now considered the primary source of energy for IoT devices. Remote areas may face challenges due to limited energy resources used by batteries ecological barriers, shortened lifespan, battery maintenance.

Energy Harvesting $(\mathrm{EH})$ is considered an effective solution to overcome all these problems. EH is the mechanism by which energy is obtained and stored for IoT devices. Two different EHs can use the following energy sources: One is external energy and the other is environmental energy. Environmental EH sources typically reside within the environment and do not require external energy data [25]. These includes energy sources such as photovoltaic (PV) energy [26, 27], vibration energy (VE) [28, 29], thermal energy [30, 31], and radio frequency (RF) [32]. The hybrid energy harvesting system (HEHS) plays an important role due to the energy density of the surrounding energy in the field of IoT [33]. More than two energy sources are included in HEHS that can increase performance and system efficiency [34]. The most popular form of HEHS for IoT devices involves the development process of microelectromechanical systems (MEMS). This is referred to as a miniature or miniature device to extract energy [35, 36].

Thermoelectric, thermo-photovoltaic, piezoelectric, and microbial fuel cells are the four types of minute-scale power machines studied in this research. Micro-scale energy converters' great potential to power IoT tinny devices in smart city applications, low power energy harvesting system and Its research trend as well as the obstacles and limits they encounter, are examined in the first and second sections of the paper. After that, for each gadget, the methodology performance analysis and studies of the highest achievements accomplished throughout the one and half decade 20052020 for the future potential of energy harvesting technology to supply power to standalone IoT devices are provided.

\section{2- Smart City Scenario Energy Harvesting}

Energy Harvesting is the method of converting ambient energy into electrical energy (EH). Several strategies have been developed throughout the years to apply this principle to a variety of sources. An EH system is made up of numerous interconnected subsystems, one of which is power production, which is responsible for powering IoT devices. Over the last decade, efficient energy harvesting for low-power devices has been achieved from sources such as thermal, solar, wind, radiofrequency, sound, and others. Figure 1 depicts an energy harvesting workflow based on light, temperature, motion, electromechanical, and electromagnetic fields. Figure 2 depicts many types of systems for various energy harvesting strategies, such as from AC and DC sources.

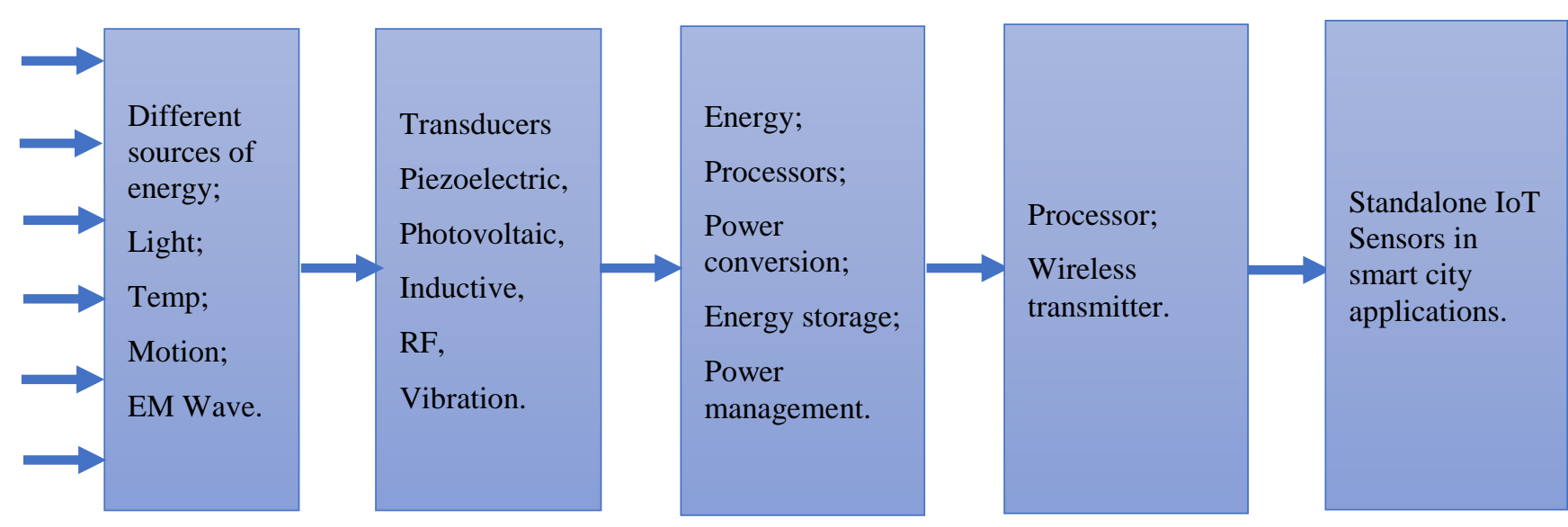

Figure 1. A workflow of energy harvesting to power IoT tinny devices in smart city application.

\section{3- Sustainability of the Internet of Things}

The successful use of spectra becomes important as the IoT becomes more prevalent. In addition, the advancement of low-cost high-end 5G mobile technology has made it possible for broadband content (such as video/multimedia) to be produced and received. Although smartphone users have a large range of advanced mobile devices, battery life is one 
of the key barriers to receiving multimedia content. One of the main reasons for user frustration is battery life limitations on high-end cell phones. With a focus on the latest developments in energy harvesting, particularly in the field of RF (RF) energy harvesting, permanent IoT operations can be realized.

\section{4- Low Power Energy Harvesting System and Its Research Trend}

Energy infrastructure systems have made significant progress. The calculated features of each energy supplier are built into the IoT sensor and can improve their capabilities of energy requirements. To overcome the energy crisis, it is necessary to develop energy harvesting system, which is a connection between IoT devices and self-powered functions. Many researchers have developed a variety of technologies to mitigate future and existing energy demand.

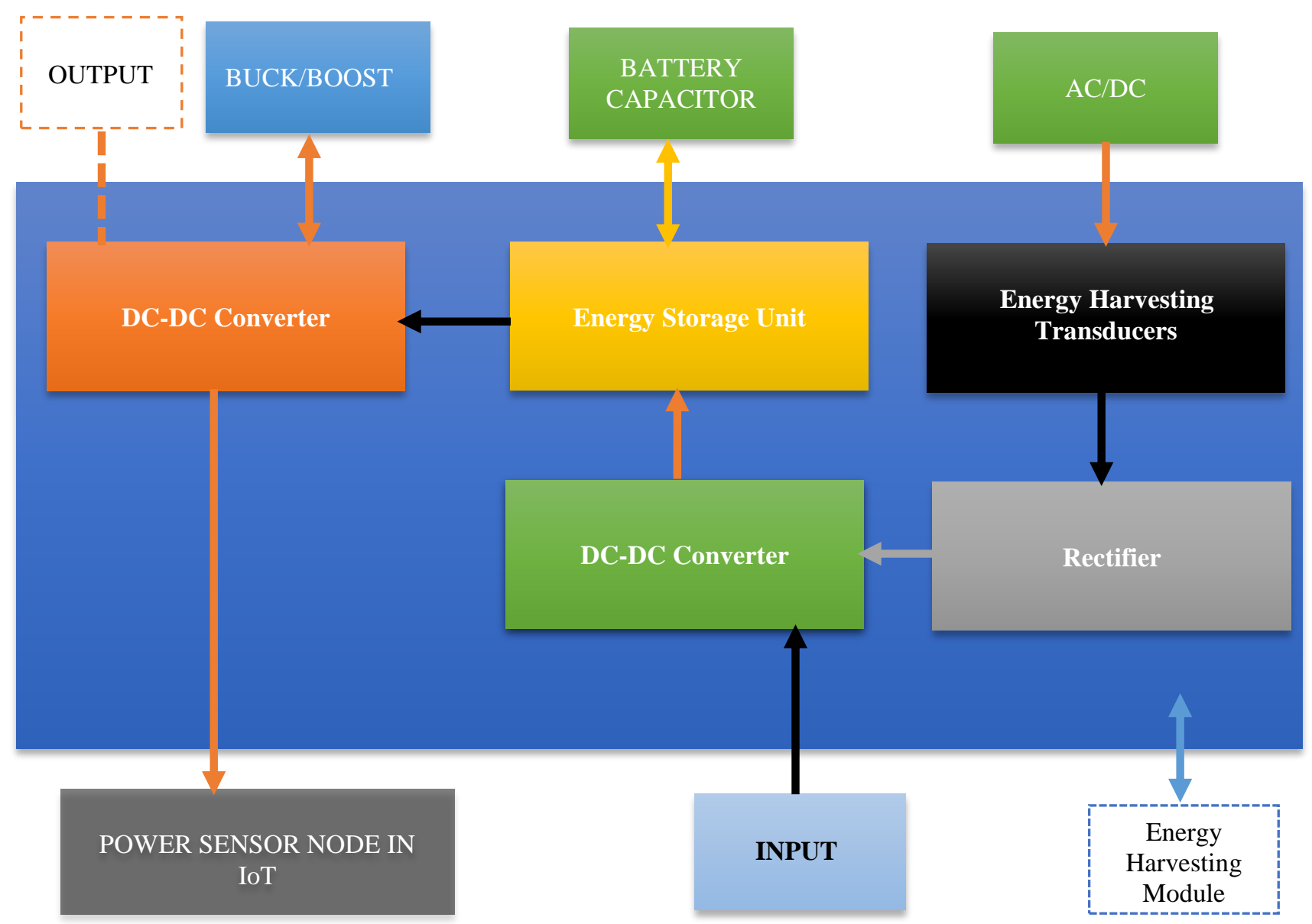

Figure 2. A Signal flow diagram for various energy harvesting strategies.

Between 1821 and 1956, the basic principles of energy harvesting were developed. The key EH timeline, showing that the EH drift started in 1821, is shown in Figure 3 when the thermoelectric effect was identified by Thomas Johann Seeback. The thermoelectric effect was studied by Thomas Johann Seebeck in 1821, and Leopoldo Nobili and Macedonio Meloni later discovered the thermopile structure in the mid-19th century. Low-cost generators with stable energy sources and fixed blocks that are easy to scale and can conduct heating and cooling processes are thermoelectric generators.

Alexander Edmond Becquerel discovered the PV effect in 1839 (PV). In addition, Pierre Curie discovered the piezoelectric effect with Jack Curie in 1880. Burnett Cohen invented Fuel Cell Microorganisms in 1931 (MFC). William C Brown established the RF concept in 1950 and later 1956 Henry Colm continued with certain modification. In the mid-1990s, when researchers concentrated more on self-sufficient energy systems for electronic devices to provide electricity for standalone devices, the popular EH movement began.

Kymissis et al. invented a piezoelectric transducer that can be stored in sports shoes. This is the first instance of an ecological energy harvester for mobile device operation [37]. Since then, integrated circuit power consumption has continued to decline, and research has made tremendous strides and gained funding. This has further extended the scope of research on self-powered electronic products, such as devices such as wireless modules and replacement batteries [38]. Storage space and processing efficiency continue to increase due to the rapid growth of cloud technology, and manufacturing and production costs continue to decrease. Over the last few decades, sensor usage has increased significantly due to increased deployment costs. By the end of 2030, fifty to hundred billion IoT devices will be 
connected to the Internet using a variety of information technologies. Figure 4 shows the projected increase in the world's population and the expected increase in the number of smart devices connected to the Internet from 2009 to 2030 . By the end of 2030, the number of smart devices is expected to reach 50 billion and the world population is estimated to reach 8.5 billion. More importantly, per person smart devices could increase from two in 2018 to about ten in 2030 .

\section{1}

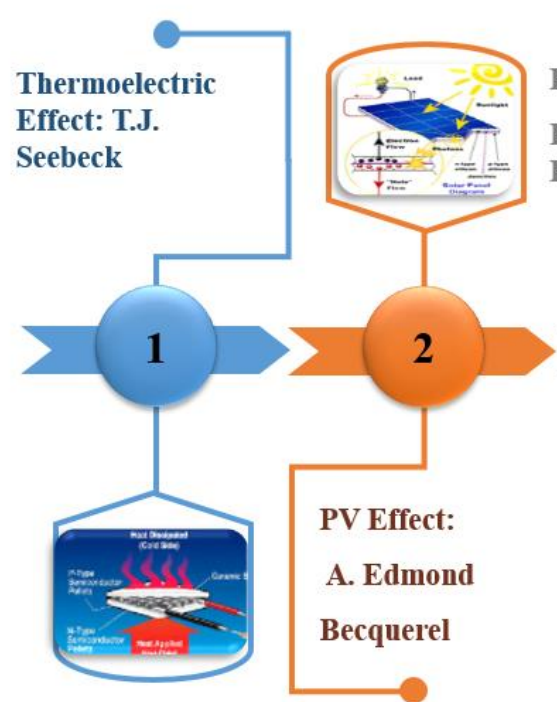

1839
1880

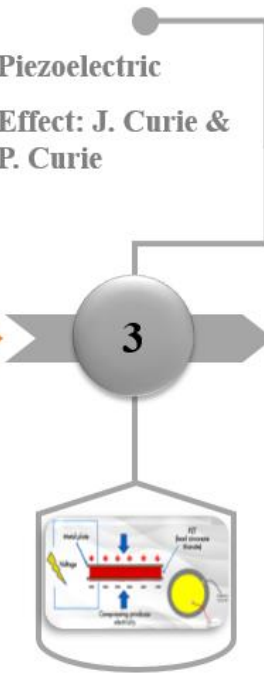

Effect: J. Curie \& Curie

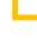

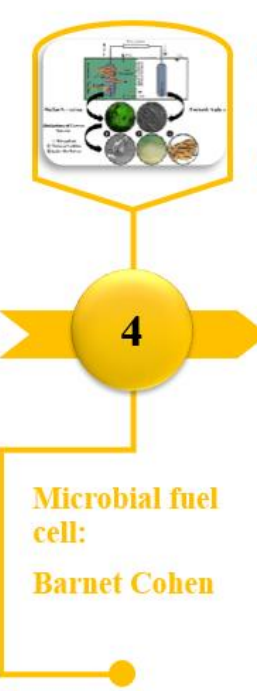

1931
1950

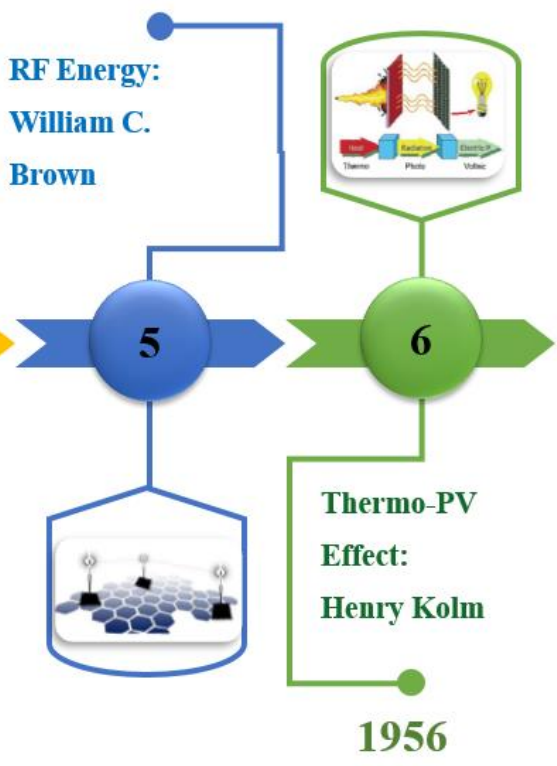

Figure 3. Milestone of energy harvesting timeline during 1821 to $1956[32,36]$.

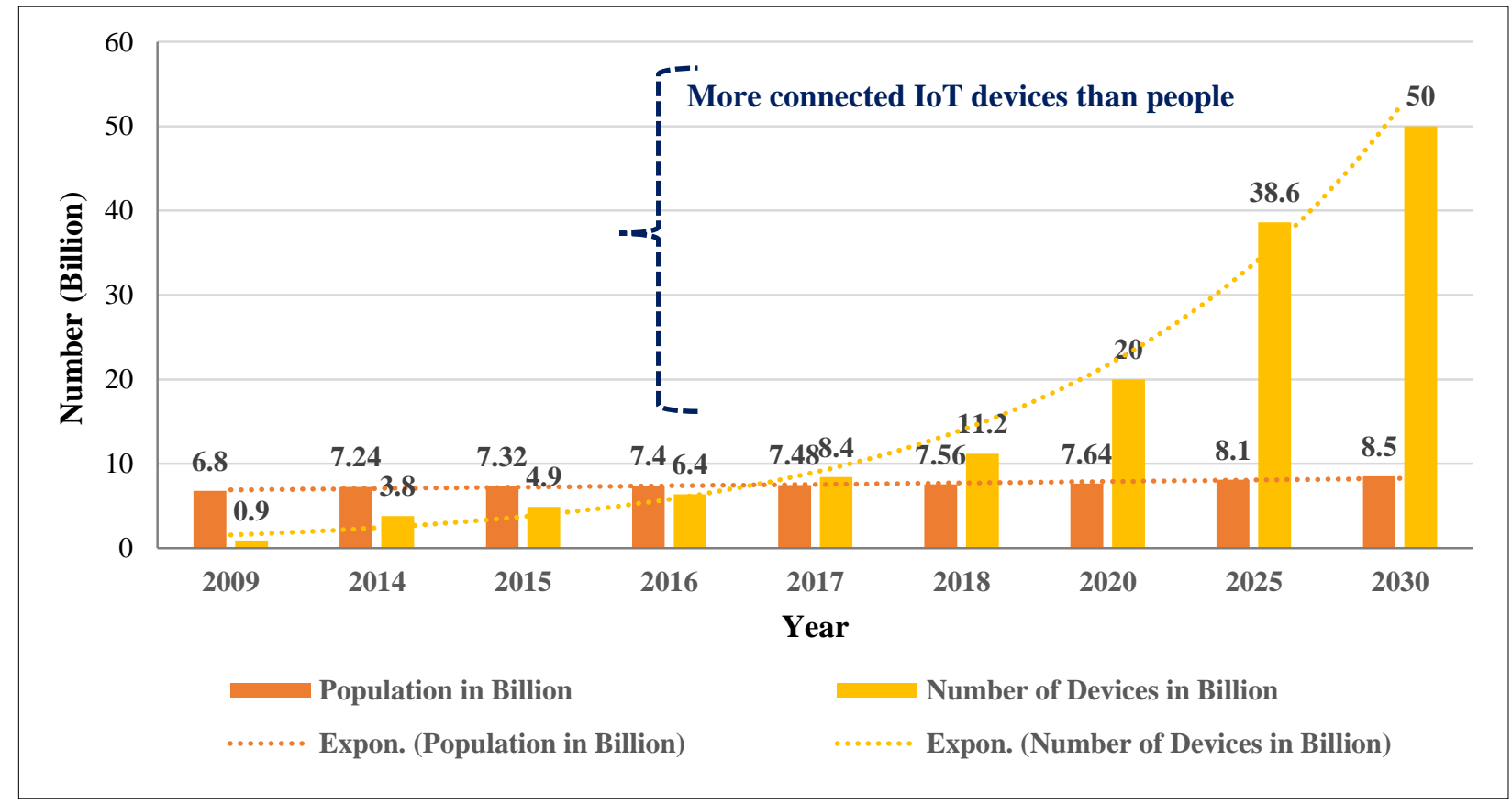

Figure 4. Number of internet of things (IoT) connected devices worldwide vs. population.

\section{5- Standalone Micro-scale Electricity Harvester to Meet the Requirement of IoT Devices}

Even in remote locations without the need for large storage components for powering IoT applications, small standalone power harvester sizes such as thermoelectric, piezoelectric, and microbial fuel cells are very useful. In addition, this energy removal unit, as shown in Figure 5, also offers many additional advantages that may draw more attention to the system's feasibility.

For example, thermoelectric generators use the heat energy of an unused environment or humans body heat to convert directly into potential differences. Today, burners are also absorbed by thermoelectric devices as an alternative heat source. Energy harvesting technology $(\mathrm{ETH})$ can turn environmental energy into electrical energy for IoT applications such as solar energy, thermal energy, vibration energy, and RF energy. As shown in Figure 2, in its simplest form, a 
complete energy harvesting device includes three main components: a transducer, an interface circuit, and a load. The use case and the energy supply needed to decide the EHT and other electronic devices in the system to be implemented. EHT is a very attractive technology for different self-powered microsystems such as IoT edge applications, according to many researchers [39].

\section{Microbial Fuel Cell}

- Ecologically healthy

- Non-hazardous

- Exploits organic wastes

- Treats effluent water

- Hydrogen gas fuel production

- Pollution control approach

\section{Thermoelectric}

- Low coast

- Reliable energy source

- No moving Blocks

- Plain configuration

- Easily scalable

- Both heating \& coaling process

- Work for more hours

- Less noise

- Little emission

- Recycles wasted heat energy

- Healthier atmosphere

\section{Piezoelectricity}

- Small size

- Higher output voltage

- Simple mechanism

- Higher sensitive to applied voltage

- Higher frequency response

- Longer life cycle

Figure 5. Standalone micro-scale electricity harvester to meet the requirement of IoT devices.

Basically, energy harvesters need to remove at least mill watts of power from the environment of IoT edge devices. Table 1 summarizes the various EHTs and the intermittent power generated by thermoelectricity. The energy generated can cover many orders of magnitude.

Table 1. Advances in thermoelectricity generators during 2005-2020.

\begin{tabular}{lc}
\hline \multicolumn{1}{c}{ Method } & Power Density in $\mathbf{m W} / \mathbf{c m}^{\mathbf{2}}$ \\
\hline Warmth of human body & 0.0285 \\
ZnSb film screen-printed & 0.17 \\
Polymer Bi-epoxy Bi0.5 Sb1.5 Te3-epoxy & 1.23 \\
Ferromagnetic material in soft nature & 3.61 \\
MnSi1.74/Mn0.7Fe0.3Si1.68 type Multi-layer & 11.7 \\
Integration photovoltaic cell & 13.8 \\
Flexible polymer Bi2Te3 & 20 \\
Wasted heat of automobile engine & 51.13 \\
Sb2Te3 layered highly-textured & 73 \\
Tubular module & 80 \\
Heat transfer system of Micro-fluidic & 126 \\
Sb-doped n-type Mg2Si Uni-leg structure & 215 \\
Silicon combustor system & 580 \\
Uni-couple PbTe-based quantum-dot super lattice & 2200 \\
ErAs: InGaAs/(InGaAs)0.2 & 2500 \\
\hline
\end{tabular}


The power required by IoT devices and sensors is in range of 0.1 to $1 \mu \mathrm{W}$. The feasible range of dimensioncontrollable energy harvesting equipment (photovoltaic cells) for power generation has been expanded to $500 \mathrm{~mW}$. This is because IoT edge devices have a power consumption of around $100 \mu \mathrm{W} / \mathrm{cm}^{2}$. The supply and demand for self-energy will occur at various times. Effective energy transfers from harvesters to IoT edge devices actually involves temporary energy buffers (such as super capacitors) and electronics for power management.

As shown in Figure 6, there are multiple sources of environmental energy, and the corresponding harvesting techniques are also depicted. These techniques are appropriate from the perspective of the size of the IoT device or the overall simplicity of the corresponding transducer at the time of generation. It is speculated that it can be used in an IoT edge device environment by supplying current directly or from energy. These sources of light, thermal energy, radiofrequency energy from the electromagnetic spectrum or induction range, kinetic energy, motion, vibration, rotation, linear motion, etc.

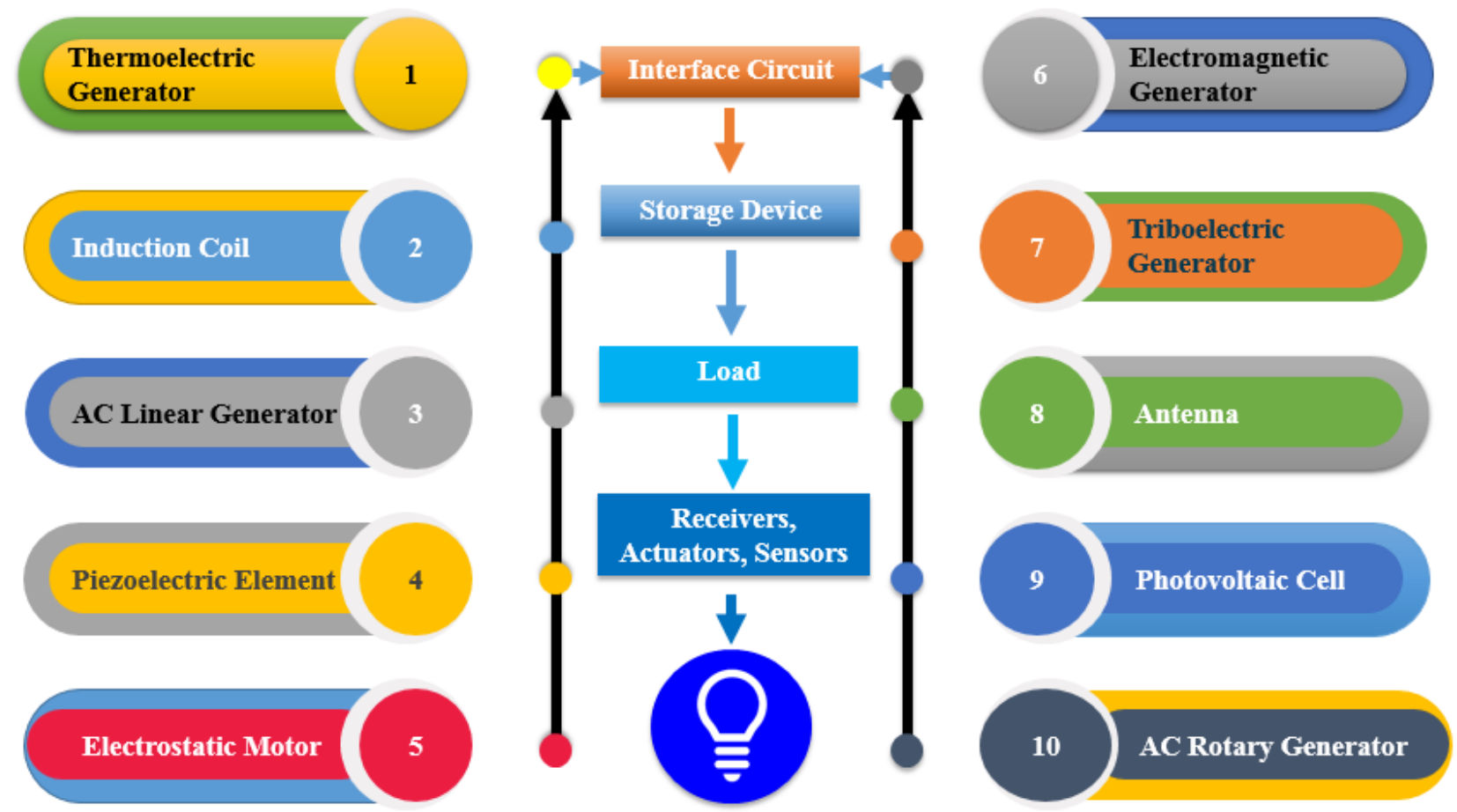

Figure 6. The ambient energy source for future standalone IoT devices and corresponding harvesting technologies.

\section{6- Future Potential of Energy Harvesting Technology to Supply Power to Standalone IoT Devices}

Some technologies' power density is not optimal and the extra cost can be an obstacle to broad implementations, but there are possibilities and multidisciplinary topics of study for the implementation of EHT. When the number of IoT devices increases, so does the number of deployed EHTs, which can improve cost savings in manufacturing. The life of EHT is about 5 years. These EHT devices can be used for more than 5 years without replacing components. Therefore, there is no need to regularly maintain the power supply unit of a remote independent IoT device. Inferred from past transistor-driven power supply units, it includes cloud computing for data processing. Assuming further reductions in energy consumption, this increases the feasibility of EHT. EHT's greatest potential for energy savings is expected to be in building automation. In the future, it can reduce the copper wire used in buildings that can reduce material, installation, and maintenance costs.

Improvements and changes in energy harvesting equipment are key points leading to more influential energy conversion appliances. A well-developed system must have powerful energy conversion capabilities. In this section, we will collect and analyze the features that each person has acquired on how to enhance the micro harvester. This is useful for comparing performance over the 15 years from 2005 to 2020 . In addition, it focuses on the best performance and the features implemented by each microsystem. Numerous improvements have been recorded over the last decade to thermoelectric micro power harvesters that can be suitable for standalone IoT devices to meet the power supply. Therefore, from 2005-2020, several important methods were considered for performance analysis, as shown in Table 1.

Today, to monitor the thermal spectral emission and photon wavelength spectrum, selective micro emitters are used as alternative filters. The heat source for the device is usually used for sunshine, heat combustion, or radionuclide radiation. High-performance thermo-optical devices are primarily designed to achieve higher energy yields with higher conversion rates. The absorption efficiency of the solar spectrum is closely related to this. The efficiency of photon 
filtering is very good for micro emitters, and the photovoltaic cell's increased bandgap energy enables more photons to be injected. However, as shown in Table 2, the improved methods adopted between 2005 and 2020 for energy transmission rates have been observed.

$\mathrm{NaNbO} 3$ nanowires $\mathrm{AlN}, \mathrm{K} / \mathrm{Na}$ ) / $\mathrm{NbO} 3, \mathrm{KNN}, \mathrm{PZT}$ nanowires, flexible barium, barium titanate (BaTiO3) Nanogenerators, flexible zinc oxide $(\mathrm{ZnO})$ nanowire KNN / manganese monoxide ( $\mathrm{Mn})$ / potassium (K) copper (C) tantalum pentoxide (T)-(KCT) materials are obviously also being checked for significant piezoelectric materials.

Table 2. Progresses of thermo-photovoltaic harvesters in 2005-2020.

\begin{tabular}{lc}
\multicolumn{1}{c}{ Method } & System efficiency in \% \\
\hline Porous medium swirling combustor reverse tube & 1.02 \\
Heat recovery with an extended outlet for exhaust & 2.1 \\
Photonic crystal thermal emitter for high temperatures & 2.5 \\
Micro-emitter heat recovery & 2.9 \\
Natural gas-fired radiant burners & 3.2 \\
Silicon micro-cavity selective emitter & 3.2 \\
Tungsten radiator combined with InGaSb & 3.2 \\
The surface of the nano-photonic hot absorber-emitter & 3.2 \\
Double-sided absorber/emitter of 2D photonic crystals & 3.74 \\
GaSb Cell Closed Quartz Tube Zinc & 3.9 \\
Heat recovery with the material of the SiC emitter wall & 4.3 \\
Containment of optics on Ge cell & 5.34 \\
Optimized Ge Cell back-side touch & 6.3 \\
Micro-reformer incorporation of Quartz & 8.1 \\
Selective absorber/emitter monolithic planer & 8.3 \\
The p-GaAs/p-Ge/n-Ge cell structure & 10.9 \\
\hline
\end{tabular}

In addition, there are some obvious enhancements such as electromagnetic piezoelectric hybrid technology, curved L-shape and changes such as anti-silicon ( $\mathrm{Si}$ ). Quality of dual-chip cantilever beams and other items of wind turbine actuators such as agitator sleeves with axial force and bimorphs and its comparisons of power density in $\mathrm{mW} / \mathrm{cm}^{3}$ are shown in Table 3.

Table 3. Growth of piezoelectricity systems during 2005-2020.

\begin{tabular}{lc}
\hline \multicolumn{1}{c}{ Method } & Power Density in $\mathbf{m W / \mathbf { c m } ^ { \mathbf { 3 } }}$ \\
\hline Cantilever straight resonant frequency & 0.16 \\
2-D mass cantilever beam & 0.24 \\
Volume optimized system & 0.31 \\
SiO2 layer on SOI & 0.42 \\
NaNbO3 nano-wire & 0.6 \\
Hybrid of electromagnetic-piezoelectric & 1.18 \\
AIN material & 1.4 \\
Curved L-shape proof mass & 1.45 \\
Blender casing with axial force & 1.51 \\
K/Na/NbO3 composite KNN material & 1.62 \\
PZT nano-wire & 2.8 \\
Si proof mass of unimorph cantilever & 3.272 \\
Flexible BaTiO3 nano-generator & 7 \\
Flexible ZnO nano-wire nano-generator & 11 \\
12 bomorph actuators on windmill & 17 \\
Bonding of bulk PZT on SOI & 28.86 \\
Four doubly clamped beam & 2000 \\
KNN/Mn/KCT material & 10000 \\
\hline & \\
\hline
\end{tabular}


Table 4 compares the methodological performance achieved over the last few years by microbial fuel cell systems using different techniques and methods.

Table 4. Trends of microbial fuel cell evolution between 2005-2020.

\begin{tabular}{lc}
\hline \multicolumn{1}{c}{ Method } & Power Density in $\boldsymbol{W / \mathbf { m } ^ { 2 }}$ \\
\hline Activated carbon air cathode & 1.22 \\
The sponge of carbon nano-tube/electrode & 1.24 \\
Increased NaCI concentration & 1.33 \\
Heat-acid treated fiber brushes & 1.37 \\
PANI-ERGNO/CC anode & 1.39 \\
Geobacter sulfur reducens/reduced anode size & 1.88 \\
Therapy with carbon cloth anode/ammonia gas & 1.97 \\
The catalyst of carbon brush anode/FePc cathode & 2.11 \\
Anode treated with Nitric acid & 2.07 \\
Felts of graphene-coated stainless steel fiber anode & 2.14 \\
Buffer solution of fabric electrode assemblies/bicarbonate & 2.77 \\
Felt electrodes of Shewanella oneidensis & 3.00 \\
DSP10/graphite Cathodic electron acceptor & 3.99 \\
Permanganate surface field combinations of anode-to-cathode & 6.86 \\
\hline
\end{tabular}

This study analyzed the best performance over the past decade of four types of small energy harvesters: thermoelectric, thermoelectric, piezoelectric, and microbial fuel cells. The materials or techniques applied, the beneficial or relevant factors, and their subsequent effects are shown in Table 5.

Table 5. Breakthrough performance achieved during 2005-2020 by micro-scale power devices.

\begin{tabular}{|c|c|c|c|}
\hline & Materials/strategies applied & Advantageous & Obtained results \\
\hline $\begin{array}{l}\text { Thermoelectric } \\
\text { [39] }\end{array}$ & $\begin{array}{l}\text { (InGaAs) } 0.8 \text { / ErAs:InGaAs } \\
\text { (InAIAs) } 0.2\end{array}$ & $\begin{array}{l}\text { Lower scrounging electrical loss, Thermal } \\
\text { conductivity reduced, Increased Seebeck coefficient } \\
\text { Stable Thermodynamically super lattice, }\end{array}$ & $\begin{array}{c}2500 \mathrm{~mW} / \mathrm{cm}^{2} \text { at } \\
3.5 \mathrm{~V}\end{array}$ \\
\hline $\begin{array}{l}\text { Thermo- } \\
\text { photovoltaic [40] }\end{array}$ & p-GaAs/ n-Ge cell /p-Ge/ & $\begin{array}{l}\text { Allowing greater absorbency of the light source, } \\
\text { compact cell layers with band-gap energy-optimized }\end{array}$ & $10.9 \%$ \\
\hline Piezoelectric [41] & KNN/Mn/KCT Material & $\begin{array}{l}\text { Along with enhanced densification. } \\
\text { Lead-free content Elevated Curie temperature, } \\
\text { Piezoelectric coefficient, and electromechanical } \\
\text { coupling element }\end{array}$ & $10,000 \mathrm{~mW} / \mathrm{cm}^{3}$ \\
\hline $\begin{array}{l}\text { Microbial fuel cell } \\
\text { [42] }\end{array}$ & $\begin{array}{l}\text { Surface field combinations of } \\
\text { anode-to-cathode }\end{array}$ & $\begin{array}{l}\text { Smooth electrons with reduced internal resistance } \\
\text { flow from anode to cathode }\end{array}$ & $\begin{array}{c}\quad 6.86 \mathrm{~W} / \mathrm{m}^{2} \\
\text { At } 2.62 \mathrm{~mA} / \mathrm{cm}^{2}\end{array}$ \\
\hline
\end{tabular}

\section{7- Results and Discussions}

From 2005 to 2020, this research looked at how to improve the performance of four different types of micro-scale energy harvesting devices in order to supply power to IoT sensors: thermoelectric, thermo-photovoltaic, piezoelectric, and microbial fuel cells. Table 5 shows the breakthrough successes as determined by the methodological performance analysis in the previous section. Table lists the materials or tactics used, as well as any beneficial or important factors, along with the related results. Thermoelectric power harvester with (InGaAs)0.8 / ErAs: InGaAs (InAIAs)0.2 materials have achieved $2500 \mathrm{~mW} / \mathrm{cm}^{2}$ at $3.5 \mathrm{~V}$. To perform better powering in thermoelectric power generation, more compact heat-to-power conversion systems are necessary, which include the use of more thermocouples, higher electron mobility, and lower heat loss from the device. More metal hybridization and heat recovery technologies should be implemented as well.

The small-scale thermo-photovoltaic harvester's development is primarily focused on heat and photon energy management throughout its module. From 2005 to 2020, this module has seen a considerable boost in energy transfer efficiency of up to $10.9 \%$ by implementing the attached cell structure of p-GaAs/ n-Ge cell /p-Ge. Because the thermophotovoltaic generator is a promising tri-generation voltage transducer, additional efforts should be made to improve energy conversion efficiency through efficient thermal management. Improvements in this energy transducer are moving at a rapid pace, as seen by the numerous studies reported throughout the ten years examined. This signals a positive 
response and a bright future for this little thermo-photovoltaic tri-generator, as it has sparked numerous research projects in the sector.

The tiny piezoelectric system, on the other hand, is a developing MEMS power harvester with a small dimension. A study of lead-free materials $\mathrm{KNN} / \mathrm{Mn} / \mathrm{KCT}$ material found $10,000 \mathrm{~mW} / \mathrm{cm}^{3}$ increase in volumetric power density of piezoelectricity. To achieve outstanding piezoelectric performance, several factors such as thin-film piezo materials, small device dimensions, strain effects, micro-beam architectures, and maximized cantilever distances necessitate rectifications. In order to upgrade low-frequency piezoelectric devices to higher oscillation resonant frequencies with greater band operations, more efforts should be made in the area of frequency up- conversions. The resulting power density and overall energy transduction can be boosted in this manner.

Meanwhile, in the last ten years, the bio-electro chemical-based microbial fuel cell energy converter has seen a peak in growth. An attempt to use a phosphate buffer with a reduced anode-to-cathode area ratio revealed a phenomenal anodic power density of up to $6.86 \mathrm{~W} / \mathrm{m}^{2}$ demonstrating the system's capabilities. As a result, additional new strategies for increasing the power provided by small microbial fuel cells while also increasing their potential for treating polluted effluents and organic wastes should be identified.

\section{8- Conclusion}

This study provides a comprehensive analysis of a wide range of promising environmental energy harvesting techniques to power standalone IoT-equipped sensor. All four types of energy harvesting microdevices show positive results in the methodology performance analysis described in this research. The powerful potential associated with energy harvesting has attracted the attention of various stakeholders involved in its design and implementation. Technology that meets the energy needs of the next 50 billion IoT devices by 2030. In this work, we have developed a comprehensive classification scheme for energy harvesting technologies to meet the power requirements of independent IoT devices. More specifically, it focuses on energy harvesting technology by using our surroundings' ambient and external resources to generate energy for independent IoT devices. Smart cities and the IoT herald a new era of urban life, increasing the safety, habitability, and comfort of citizens and enabling high-tech companies to achieve more efficient and smart urban services and management. Recently, extensive research has been conducted to investigate the unique power harvesting technology for IoT applications. Designing energy harvesters for stand-alone IoT devices, with traditional thermoelectricity, piezoelectric, thermo-photovoltaic and microbial fuel cell devices have been analyzed. It also examined the levels of energy harnessing, collective structure, the estimate of the energy levels obtained, and the capacity of a harvester of each class and subclass. In addition, a thorough study of the various EH models were carried out to evaluate the possible energy cycles to tackle the problems associated with the disrupted power supply for powering IoT nodes. Furthermore, in terms of environmental considerations, active attempts to find new techniques to manage heavy metal contaminated wastewaters while generating electricity may improve the microbial fuel cell system's capability. To provide improved capabilities in the future, more advanced approaches will be necessary to raise the energy conversion efficiency and generated output power from these four micro-systems.

\section{9- Declarations}

\section{9-1-Data Availability Statement}

The data presented in this study are available in article.

\section{9-2- Funding}

The author received no financial support for the research, authorship, and/or publication of this article.

\section{9-3- Conflicts of Interest}

The author declares that there is no conflict of interests regarding the publication of this manuscript. In addition, the ethical issues, including plagiarism, informed consent, misconduct, data fabrication and/or falsification, double publication and/or submission, and redundancies have been completely observed by the author.

\section{0- References}

[1] Paradiso, Joseph A., and Thad Starner. "Energy Scavenging for Mobile and Wireless Electronics.” IEEE Pervasive Computing 4, no. 1 (2005): 18-27. doi:10.1109/MPRV.2005.9.

[2] Mishra, Deepak, Swades De, Soumya Jana, Stefano Basagni, Kaushik Chowdhury, and Wendi Heinzelman. "Smart RF Energy Harvesting Communications: Challenges and Opportunities.” IEEE Communications Magazine, (2015), 70-78. doi:10.1109/MCOM.2015.7081078.

[3] Tzounis, Antonis, Nikolaos Katsoulas, Thomas Bartzanas, and Constantinos Kittas. "Internet of Things in Agriculture, Recent Advances and Future Challenges.” Biosystems Engineering 164 (2017): 31-48. doi:10.1016/j.biosystemseng.2017.09.007. 
[4] Alavi, Amir H., Pengcheng Jiao, William G. Buttlar, and Nizar Lajnef. "Internet of Things-Enabled Smart Cities: State-of-theArt and Future Trends." Measurement: Journal of the International Measurement Confederation 129 (2018): 589-606. doi:10.1016/j.measurement.2018.07.067.

[5] Dhillon, Parwinder Kaur. "Elliptic Curve Cryptography for Real Time." 2016 5th International Conference on Wireless Networks and Embedded Systems (WECON), 2016.

[6] Patil, Neha, Shrikant Ambatkar, and Sandeep Kakde. "IoT Based Smart Surveillance Security System Using Raspberry Pi." Proceedings of the 2017 IEEE International Conference on Communication and Signal Processing, ICCSP 2017, $344-348$. doi:10.1109/ICCSP.2017.8286374.

[7] Sruthy, S., and Sudhish N. George. "WiFi Enabled Home Security Surveillance System Using Raspberry Pi and IoT Module." 2017 IEEE International Conference on Signal Processing, Informatics, Communication and Energy Systems, SPICES 2017, 2017. doi:10.1109/SPICES.2017.8091320.

[8] Mubeen, Saad, Pavlos Nikolaidis, Alma DIdic, Hongyu Pei-Breivold, Kristian Sandstrom, and Moris Behnam. "Delay Mitigation in Offloaded Cloud Controllers in Industrial IoT.” IEEE Access, 2017. doi:10.1109/ACCESS.2017.2682499.

[9] Bardwell, Michael, Jason Wong, Steven Zhang, and Petr Musilek. "Design Considerations for Iot-Based Pv Charge Controllers." Proceedings - 2018 IEEE World Congress on Services, SERVICES, 2018. doi:10.1109/SERVICES.2018.00043.

[10] Sherly, J, and D Somasundareswari. "Internet of Things Based Smart Transportation Systems.” International Research Journal of Engineering and Technology, (2015): 1207-1210.

[11] Rathore, M. Mazhar, Awais Ahmad, Anand Paul, and Uthra Kunathur Thikshaja. "Exploiting Real-Time Big Data to Empower Smart Transportation Using Big Graphs.” Proceedings - 2016 IEEE Region 10 Symposium, TENSYMP, (2016): 135-139. doi:10.1109/TENCONSpring.2016.7519392.

[12] Zeng, Wei, Lin Shu, Qiao Li, Song Chen, Fei Wang, and Xiao Ming Tao. "Fiber-Based Wearable Electronics: A Review of Materials, Fabrication, Devices, and Applications.” Advanced Materials, (2014): 5310-5336. doi:10.1002/adma.201400633.

[13] R. Jayanthi, and S.T. Rama. "IOT Based Smart Energy Tracking System" International Journal of MC Square Scientific Research 9(1), (2017): 98-108. doi:10.20894/ijmsr.117.009.001.012.

[14] Mois, George, Silviu Folea, and Teodora Sanislav. "Analysis of Three IoT-Based Wireless Sensors for Environmental Monitoring." IEEE Transactions on Instrumentation and Measurement 66(8), (2017): $2056-2064$. doi:10.1109/TIM.2017.2677619.

[15] Alvarez-Campana, Manuel, Gregorio López, Enrique Vázquez, Víctor Villagrá, and Julio Berrocal. "Smart CEI Moncloa: An IoT-Based Platform for People Flow and Environmental Monitoring on a Smart University Campus." Sensors 17, no. 12 (December 8, 2017): 2856. doi:10.3390/s17122856.

[16] Koubaa, Anis, and Basit Qureshi. "DroneTrack: Cloud-Based Real-Time Object Tracking Using Unmanned Aerial Vehicles over the Internet.” IEEE Access, (2018). doi:10.1109/ACCESS.2018.2811762.

[17] Celesti, Antonio, Antonino Galletta, Lorenzo Carnevale, Maria Fazio, Aime Lay-Ekuakille, and Massimo Villari. "An IoT Cloud System for Traffic Monitoring and Vehicular Accidents Prevention Based on Mobile Sensor Data Processing.” IEEE Sensors Journal 18(12), (2018): 4795-4802. doi:10.1109/JSEN.2017.2777786.

[18] Sukode, Sagar, and Shilpa Gite. "Vehicle Traffic Congestion Control \& Monitoring System in IoT." International Journal of Applied Engineering Research 10(8), (2015): 19513-19523.

[19] Zamora-Izquierdo, Miguel A., José Santa, Juan A. Martínez, Vicente Martínez, and Antonio F. Skarmeta. "Smart Farming IoT Platform Based on Edge and Cloud Computing." Biosystems Engineering, (2019): 4-17. doi:10.1016/j.biosystemseng.2018.10.014.

[20] Jawad, Haider Mahmood, Rosdiadee Nordin, Sadik Kamel Gharghan, Aqeel Mahmood Jawad, and Mahamod Ismail. "EnergyEfficient Wireless Sensor Networks for Precision Agriculture: A Review.” Sensors (Switzerland), 2017. doi:10.3390/s17081781.

[21] Riaz, M. H., U. Rashid, M. Ali, and L. Li. “Internet of Things Based Wireless Patient Body Area Monitoring Network.” 2017 IEEE International Conference on Internet of Things (iThings) and IEEE Green Computing and Communications (GreenCom) and IEEE Cyber, Physical and Social Computing (CPSCom) and IEEE Smart Data (SmartData) (June 2017). doi:10.1109/ithings-greencom-cpscom-smartdata.2017.180.

[22] Morgan, Jacob. "A Simple Explanation of "The Internet of Things." Forbes, 2014. Available online: http://www.forbes.com/sites/jacobmorgan/2014/05/13/simple-explanation-internet-things-that-anyone-can-understand/ (accessed on August 2021).

[23] Babar, Muhammad, Ataur Rahman, Fahim Arif, and Gwanggil Jeon. "Energy-Harvesting Based on Internet of Things and Big Data Analytics for Smart Health Monitoring." Sustainable Computing: Informatics and Systems 20 (2018): 155-64. doi:10.1016/j.suscom.2017.10.009. 
[24] Kortuem, Gerd, Fahim Kawsar, Vasughi Sundramoorthy, and Daniel Fitton. "Smart Objects as Building Blocks for the Internet of Things.” IEEE Internet Computing, 2010. doi:10.1109/MIC.2009.143.

[25] Shaikh, Faisal Karim, and Sherali Zeadally. "Energy Harvesting in Wireless Sensor Networks: A Comprehensive Review." Renewable and Sustainable Energy Reviews 55 (2016): 1041-54. doi:10.1016/j.rser.2015.11.010.

[26] Ryu, Hanjun, Hong Joon Yoon, and Sang Woo Kim. "Hybrid Energy Harvesters: Toward Sustainable Energy Harvesting." Advanced Materials, 2019. doi:10.1002/adma.201802898.

[27] Lee, Ju Hyuck, Jeonghun Kim, Tae Yun Kim, Md Shahriar Al Hossain, Sang Woo Kim, and Jung Ho Kim. "All-in-One Energy Harvesting and Storage Devices.” Journal of Materials Chemistry A, (2016): 7983-7999. doi:10.1039/c6ta01229a.

[28] Turconi, Giovanna, Marianna Guarcello, Federica Cignoli, Stefania Setti, Rosella Bazzano, Carla Roggi, and Laura Maccarini. "Eating Habits and Behaviors, Physical Activity, Nutritional and Food Safety Knowledge and Beliefs in an Adolescent Italian Population." Journal of the American College of Nutrition. March, 2008. doi:10.1080/07315724.2008.10719672.

[29] Drezet, C., N. Kacem, and N. Bouhaddi. "Design of a Nonlinear Energy Harvester Based on High Static Low Dynamic Stiffness for Low Frequency Random Vibrations.” Sensors and Actuators, A: Physical, 2018. doi:10.1016/j.sna.2018.09.046.

[30] Champagne, C., and L. Weiss. "Performance Analysis of a Miniature Free Piston Expander for Waste Heat Energy Harvesting." Energy Conversion and Management 76 (2013): 883-92. doi:10.1016/j.enconman.2013.08.045.

[31] Kraemer, D, G Chen, Y Cui, and A Electrochemical. "Harvesting Low-Grade Heat Energy,” (2014).

[32] Tran, Le Giang, Hyouk Kyu Cha, and Woo Tae Park. "RF Power Harvesting: A Review on Designing Methodologies and Applications." Micro and Nano Systems Letters 5, no. 1 (2017). doi:10.1186/s40486-017-0051-0.

[33] Lee, In, and Kyoochun Lee. “The Internet of Things (IoT): Applications, Investments, and Challenges for Enterprises.” Business Horizons 58, no. 4 (2015): 431-40. doi:10.1016/j.bushor.2015.03.008.

[34] Chandrasekaran, Sundaram, Chris Bowen, James Roscow, Yan Zhang, Dinh Khoi Dang, Eui Jung Kim, R. D.K. Misra, Libo Deng, Jin Suk Chung, and Seung Hyun Hur. "Micro-Scale to Nano-Scale Generators for Energy Harvesting: Self Powered Piezoelectric, Triboelectric and Hybrid Devices.” Physics Reports 792 (2019): 1-33. doi:10.1016/j.physrep.2018.11.001.

[35] Shi, Qiongfeng, Tianyiyi He, and Chengkuo Lee. "More than Energy Harvesting - Combining Triboelectric Nanogenerator and Flexible Electronics Technology for Enabling Novel Micro-/Nano-Systems." Nano Energy 57 (2019): 851-71. doi:10.1016/j.nanoen.2019.01.002.

[36] Selvan, Krishna Veni, and Mohamed Sultan Mohamed Ali. "Micro-Scale Energy Harvesting Devices: Review of Methodological Performances in the Last Decade." Renewable and Sustainable Energy Reviews 54 (2016): $1035-47$. doi:10.1016/j.rser.2015.10.046.

[37] Kymissis, John, Clyde Kendall, Joseph Paradiso, and Neil Gershenfeld. "Parasitic Power Harvesting in Shoes." International Symposium on Wearable Computers, Digest of Papers 1998-October (1998): 132-39. doi:10.1109/ISWC.1998.729539.

[38] Roundy, S., and P. K. Wright. "A Piezoelectric Vibration Based Generator for Wireless Electronics." Smart Materials and Structures, (2004). doi:10.1088/0964-1726/13/5/018.

[39] Lv, Song, Zuoqin Qian, Dengyun Hu, Xiaoyuan Li, and Wei He. "A Comprehensive Review of Strategies and Approaches for Enhancing the Performance of Thermoelectric Module.” Energies 13, no. 12 (2020): 3142. doi:10.3390/en13123142.

[40] Maghsoudi, Peyman, Javad Gholami, Ayat Gharehghani, and Shahab Moshari. "Evaluation of Thermo Photovoltaic Performance through Aluminum-Fueled Combustor with Partially Porous Medium and Different Geometric Cross-Sections." Energy Conversion and Management 247, no. 1 (2021): 114765. doi:10.1016/j.enconman.2021.114765.

[41] Machado, Lucas Q., Danill Yurchenko, Junlei Wang, Giacomo Clementi, Samuel Margueron, and Ausrine Bartasyte. "MultiDimensional Constrained Energy Optimization of a Piezoelectric Harvester for E-Gadgets.” IScience 24, no. 7 (2021): 102749. doi:10.1016/j.isci.2021.102749.

[42] Fadzli, Fatin Syahirah, Showkat Ahmad Bhawani, and Rania Edrees Adam Mohammad. "Microbial Fuel Cell: Recent Developments in Organic Substrate Use and Bacterial Electrode Interaction." Journal of Chemistry 2021 (2021): 1-16. doi:10.1155/2021/4570388. 